\title{
Schumann resonance transients and the search for gravitational waves
}

\author{
Z.K. Silagadze* \\ Budker Institute of Nuclear Physics and Novosibirsk State University, Novosibirsk 630 090, Russia
}

\begin{abstract}
Schumann resonance transients which propagate around the globe can potentially generate a correlated background in widely separated gravitational wave detectors. We show that due to the distribution of lightning hotspots around the globe these transients have characteristic time lags, and this feature can be useful to further suppress such a background, especially in searches of the stochastic gravitational-wave background. A brief review of the corresponding literature on Schumann resonances and lightnings is also given.
\end{abstract}

\section{INTRODUCTION}

The detection of gravitational-wave signals from inspiralling binary black holes by the Advanced Laser Interferometer Gravitational-Wave Observatory (LIGO) [1 3] opens a new era in observational astrophysics and thus is of paramount importance. Recent three-detector (including Virgo) observation of the gravitational wave GW170814 [4], enabling a new class of phenomenological tests of gravity, as well as unprecedented joint gravitational and electromagnetic observation of a neutron star merger [5] are further solid confirmations that this new era indeed has come.

As the gravitational-wave detectors are extremely sensitive instruments they are prone to many sources of noise that need to be identified and removed from the data. An impressive amount of efforts were undertaken by the LIGO collaboration to ensure that GW150914 signal was really the first detection of gravitational waves with all transient noise backgrounds being under a good control [6 8].

Correlated magnetic fields from Schumann resonances constitute a well known potential source of correlated noise in gravitational waves detectors 9 -13. Such correlated noise may be a serious limiting factor in a search of stochastic gravitational-wave background which is expected to be created due to many independent, uncorrelated, not individually resolvable astrophysical and cosmological sources.

For short duration gravitational-wave transients, like the gravitational-wave signals observed by LIGO, Schumann resonances are not considered as significant noise sources because the magnetic field amplitudes induced by even strong remote lightning strikes usually are of the order of a picotesla, too small to produce strong signals in the LIGO gravitational-wave channel [6].

However, a recent study of short duration magnetic field transients, that were coincident in low-noise magnetometers in Poland and Colorado, revealed that there was about 2.3 coincident events per day where the amplitude of one of the pulses exceeded $200 \mathrm{pT}$ [14]. This finding indicates that there would be a few such events per day that would appear simultaneously at the LIGO gravitational-wave detector sites and could move the test masses by the amplitude that is comparable to the amplitude of the real GW150914 event [14].

Although it is highly unlikely that under the adopted data analysis strategy this relatively rare strong Schumann resonance transients can imitate a real gravitational wave signal, they certainly deserve a further study.

\section{SCHUMANN RESONANCES}

Schumann resonances are global electromagnetic resonances in the Earth-ionosphere cavity [15, 16]. The electromagnetic waves in the extremely low frequencies (ELF) range $(3 \mathrm{~Hz}$ to $3 \mathrm{kHz})$ are mostly confined in this spherical cavity and their propagation is characterized by very low attenuation which in the $5 \mathrm{~Hz}$ to $60 \mathrm{~Hz}$ frequency range is of the order of $0.5-1 \mathrm{db} / \mathrm{Mm}$. Schumann resonances are eigenfrequencies of the Earth-ionosphere cavity. They are constantly excited by lightning discharges around the globe. While individual lightning signals below $100 \mathrm{~Hz}$ are very weak, thanks to the very low attenuation, related ELF electromagnetic waves can be propagated a number of times around the globe, constructively interfere for wavelengths comparable with the Earth's circumference and create standing waves in the cavity.

*Electronic address: Z.K.Silagadze@inp.nsk.su 
For the ideal Earth-ionosphere cavity, Schumann obtained the following formula for the resonance frequencies [15, 16]

$$
f_{n}=\frac{c}{2 \pi R} \sqrt{n(n+1)}
$$

where $R=6371 \mathrm{~km}$ is Earth's mean radius and $c$ is light velocity in vacuum. The observed Schumann resonance frequencies $7.8,14.8,19.8,26 \mathrm{~Hz}$ are lower than predicted by (1) because the real Earth-ionosphere cavity is not an ideal one. Note that there exists some day-night variation of the resonance frequencies, and some catastrophic events, like a nuclear explosion, simultaneously lower all the resonance frequencies by about $0.5 \mathrm{~Hz}$ due to lowering of the effective ionosphere height [17]. Interestingly, frequency decrease of comparable magnitude of the first Schumann resonance, caused by the extremely intense cosmic gamma-ray flare, was reported in [18].

Usually eight distinct Schumann resonances are reliably detected in the frequency range from $7 \mathrm{~Hz}$ to $52 \mathrm{~Hz}$. However five more were detected thanks to particularly intense lightning discharges, thus extending the frequency range up to $90 \mathrm{~Hz}[19]$.

In fact (11), as a formula describing oscillations in a spherical condenser with the small separation distance between spheres, was obtained already in 1894 by Joseph Larmor [20]. In general, Schumann resonances history is an interesting story including such names as George Fitzgerald and Nikola Tesla [20].

Phase velocity $V_{p}$ of the ELF electromagnetic wave is approximately given by the formula [16] ( $f$ is the frequency in $\mathrm{Hz}$ )

$$
V_{p}=\frac{c}{1.64-0.179 \ln f+0.0179 \ln ^{2} f},
$$

which for $f=10 \mathrm{~Hz}$ gives $V_{p}=0.75 c$.

Interestingly enough, the Schumann resonances make the Earth a natural gravitational-wave detector, albeit not very sensitive [21]. As the Earth is positively charged with respect to ionosphere, a static electric field, the so-called fair weather field is present in the earth-ionosphere cavity. In the presence of this background electric field, the infalling gravitational wave of suitable frequency resonantly excites the Schumann eigenmodes, most effectively the second Schumann resonance [21]. Unfortunately, it is not practical to turn Earth into a gravitational-wave detector. Because of the weakness of the fair weather field (about $100 \mathrm{~V} / \mathrm{m}$ ) and low value of the quality factor (from 2 to 6 ) of the Earth-ionosphere resonant cavity, the sensitivity of such detector will be many orders of magnitude smaller than the sensitivity of the modern gravitational-wave detectors.

\section{ELF FIELD TRANSIENTS}

The main source of the Schumann ELF waves are negative cloud-to-ground lightning discharges with the typical charge moment change of about $6 \mathrm{Ckm}$. On Earth, storm cells, mostly in the tropics, generate about 50 such discharges per second. They generate the background Schumann resonance field with about $1 \mathrm{pT} / \sqrt{\mathrm{Hz}}$ spectral density in the first Schumann resonance peak.

The positive cloud-to-ground atmospheric discharges have significantly higher charge moment changes of about $250 \mathrm{Ckm}$ [14]. These strong lightnings generate ELF field transients clearly visible above the Schumann resonance background noise even at at large distances.

The so-called Q-bursts are more strong positive cloud-to-ground atmospheric discharges with charge moment changes of order of $1000 \mathrm{Ckm}$. ELF pulses excited by Q-bursts propagate around the globe. At very far distances only the low frequency components of the ELF pulse will be clearly visible, because the higher frequency components experience more attenuation than the lower frequency components.

Transient luminous events, such as Sprites and Gigantic Jets, are associated to the most powerful discharges between the clouds and the ionosphere. Charge moment change in this case can reach several thousand Ckm [14]. At that the occurrence of a Sprite is usually preceded by a positive cloud-to-ground discharge.

Gigantic Jets generate ELF field pulses with very large amplitudes, visible anywhere on Earth. A Gigantic Jet near Corsica on December 12, 2009 generated the transient magnetic field that was simultaneously observed as a very loud signal in magnetometers of the Virgo and LIGO-Hanford gravitational-wave detectors, and as a somewhat smaller signal in the LIGO-Livingston magnetometer. At that this Gigantic Jet actually created a perceptible gravitationalwave signal in Virgo detector (no signal was reliably observed in the LIGO detectors although their spectrograms also do show some disturbances that are coincident with the event) [14].

Fortunately Gigantic Jets are relatively rare, from a few to a dozen per year. However it was demonstrated in [14] that relatively frequent magnetic transient events of sufficiently large amplitudes can be observed in coincidence on global distances. Therefore it can be expected that strong ELF transients, related to powerful lightnings and Q-bursts across the globe, may be potential sources of correlated background for gravitational-wave detectors. 


\section{TIME LAGS OF ELF FIELD TRANSIENTS FOR LIGO DETECTORS}

In 22] Earth's lightning hotspots are revealed in detail using 16 years of space-based Lightning Imaging Sensor observations. Information about locations of these lightning hotspots allows us to calculate time lags between arrivals of the ELF transients from these locations to the LIGO-Livingston (latitude $30.563^{\circ}$, longitude $-90.774^{\circ}$ ) and LIGOHanford (latitude $46.455^{\circ}$, longitude $-119.408^{\circ}$ ) gravitational-wave detectors.

ELF transients propagate along great circles paths and it is convenient to calculate the relevant approximate distances by haversine formula $[23,24]$

$$
d=2 R \arcsin \left(\sqrt{\sin ^{2}\left(\frac{\varphi_{2}-\varphi_{1}}{2}\right)+\cos \varphi_{1} \cos \varphi_{2} \sin ^{2}\left(\frac{\lambda_{2}-\lambda_{1}}{2}\right)}\right)
$$

where $\varphi_{1}, \varphi_{2}$ and $\lambda_{1}, \lambda_{2}$ are latitudes and longitudes of two points on the Earth's surface, respectively.

We also need ELF transient propagation velocity. This was measured in [25] using the round-the-world ELF transient signals and turned out to be [25, 26] $V_{g}=265 \pm 1 \mathrm{Mm} / \mathrm{s}$ that is about $0.88 \mathrm{c}$.

We have taken Earth's lightning hotspots from [22] with lightning flash rate densities more than about $100 \mathrm{fl} \mathrm{km}^{-2} \mathrm{yr}^{-1}$ and calculated the expected time lags between ELF transients arrivals from these locations to the LIGO detectors. The results are summarized in the Table II Note that the observed group velocity for short ELF

TABLE I: Top lightning hotspots with flash rate densities (FRD) more than about $100 \mathrm{fl} \mathrm{km}^{-2} \mathrm{yr}^{-1}$, their latitude (Lat.) and longitude (Long.) positions, distances to the LIGO-Hanford (LHO) and LIGO-Livingston (LLO) detectors in km, and expected time lags in ms.

\begin{tabular}{|c|c|c|c|c|c|}
\hline FRD/Region & Lat. & Long. & d to LHO & d to LLO & Time lag \\
\hline 232.52 South America & 9.75 & -71.65 & 6072 & 3045 & 11.5 \\
\hline 172.29 South America & 7.55 & -75.35 & 6018 & 3021 & 11.4 \\
\hline 138.61 South America & 8.85 & -73.05 & 6055 & 3035 & 11.4 \\
\hline 124.26 /South America & 5.75 & -74.95 & 6206 & 3217 & 11.3 \\
\hline 114.19 /South America & 8.45 & -74.55 & 5991 & 2981 & 11.4 \\
\hline 105.73 South America & 8.15 & -76.85 & 5867 & 2882 & 11.3 \\
\hline \hline $205.31 /$ Africa & -1.85 & 27.75 & 14123 & 12821 & 4.9 \\
\hline $176.71 /$ Africa & -3.05 & 27.65 & 14235 & 12884 & 5.1 \\
\hline $143.21 /$ Africa & -0.95 & 27.95 & 14044 & 12784 & 4.8 \\
\hline $129.58 /$ Africa & 5.25 & 9.35 & 12375 & 10673 & 6.4 \\
\hline $129.50 /$ Africa & 0.25 & 28.45 & 13951 & 12756 & 4.5 \\
\hline $127.52 /$ Africa & -1.55 & 20.95 & 13717 & 12166 & 5.9 \\
\hline $117.98 /$ Africa & 0.55 & 20.35 & 13483 & 11985 & 5.7 \\
\hline $117.19 /$ Africa & -2.45 & 26.95 & 14139 & 12783 & 5.1 \\
\hline $116.78 /$ Africa & 6.95 & 10.45 & 12290 & 10677 & 6.1 \\
\hline $112.17 /$ Africa & 0.35 & 26.65 & 13849 & 12584 & 4.8 \\
\hline \hline $143.11 /$ Asia & 34.45 & 72.35 & 10942 & 12573 & -6.2 \\
\hline $121.41 /$ Asia & 33.35 & 74.55 & 11031 & 12743 & -6.5 \\
\hline $118.81 /$ Asia & 33.75 & 70.75 & 11039 & 12605 & -5.9 \\
\hline $108.03 /$ Asia & 14.55 & 43.45 & 13018 & 13007 & 0.0 \\
\hline $104.59 /$ Asia & 33.85 & 73.25 & 10996 & 12659 & -6.3 \\
\hline $101.79 /$ Asia & 25.25 & 91.95 & 11440 & 13802 & -8.9 \\
\hline $116.76 /$ North America & 14.35 & -91.15 & 4433 & 1803 & 10.0 \\
\hline $103.23 /$ North America & 14.85 & -92.05 & 4336 & 1752 & 9.8 \\
\hline $100.63 /$ North America & 22.35 & -83.25 & 4156 & 1138 & 11.4 \\
\hline $100.24 /$ North America & 18.55 & -74.35 & 5139 & 2127 & 11.4 \\
\hline $99.39 /$ North America & 13.15 & -87.25 & 4767 & 1970 & 10.6 \\
\hline
\end{tabular}

field transients depends on the upper frequency limit of the receiver [14]. For the magnetometers used in [14] this 
frequency limit was $300 \mathrm{~Hz}$ corresponding to the quoted group velocity of about $0.88 \mathrm{c}$. For the LIGO detectors the coupling of magnetic field to differential arm motion decreases by an order of magnitude for $30 \mathrm{~Hz}$ compared to $10 \mathrm{~Hz}$ [6]. Thus for the LIGO detectors, as the ELF transients receivers, the more appropriate upper frequency limit is about $30 \mathrm{H}$, not $300 \mathrm{~Hz}$. According to (2), low frequencies propagate with smaller velocities $0.75 \mathrm{c}-0.8 \mathrm{c}$. Therefore the inferred time lags in the Table might be underestimated by about $15 \%$.

\section{CONCLUDING REMARKS}

If the strong lightnings and Q-bursts indeed contribute to the LIGO detectors correlated background when the distribution of lightning hotspots around the globe can lead to some regularities in this correlated noise. Namely, ELF transients due to lightnings in Africa will be characterized by 5-7 ms time lags between the LIGO-Hanford and LIGO-Livingston detectors. Asian lightnings lead to time lags which have about the same magnitude but the opposite sign. Lightnings in North and South Americas should lead positive time lags of about 11-13 ms, greater than the light propagation time between the LIGO-Hanford and LIGO-Livingston detectors.

We don't think that such correlated background represent a serious obstacle in the gravitational-wave search because really strong lightnings and Q-bursts are relatively rare. However, as was mentioned in [14], it will be useful to have a carefully designed low-noise magnetometers system, installed at electromagnetically quiet locations, to further mitigate such a background noise. Maybe some of such ELF transient events can be used even for calibration purposes. Namely to monitor the coupling of magnetic field to differential arm motion in the gravitational-wave detectors.

LIGO and Virgo magnetometers routinely detect Schumann resonance background signal (see, for example, [12]). The observation of individual ELF transients is, however, more complicated task because the magnetometers operate in the quite noisy magnetic environments at the LIGO and Virgo sites. Therefore, the above mentioned additional system of magnetometers will be very helpful if we wish to reliable detect all relevant global ELF transients. Additionally, this global low-noise magnetometers system can be used in the lightning studies which is by itself an interesting and important scientific topic [15].

It is usually assumed that in the far field region the horizontal electric-field and vertical magnetic field tend to zero. Recently, it was demonstrated that in reality this assumption is not always justified probably because of local inhomogeneities of the ground's conductivity [27]. As a result some of the magnetic field's energy can hide in the vertical component. This circumstance should be taken into consideration when designing ELF transients monitoring system.

Observations indicate that the background and transient activity in Schumann Resonances are linked. Namely, the intensity of the background is roughly proportional to the number of large mesoscale lightnings [28]. The mechanism of this connection is the following [28, 29]. The background signal represents the result of integration of lightnings from the ordinary late-afternoon thunderstorms at lightning hotspots. Later these thunderstorms amalgamate into late evening mesoscale convective systems which are good producers of mesoscale lightning flashes with exceptionally energetic transients. However all details of this interaction are still unknown and deserve a thorough study [29].

The suppression of the Schumann resonances related background is especially important in searches of stochastic gravitational-wave background. We hope that the fact that the Schumann resonance transients are expected to have characteristic time lags will help to reduce this background.

A special ELF transients monitoring system, if developed, will allow to investigate the above mentioned fundamental interaction between background and transient phenomena in Schumann resonances and the acquired knowledge can be used to reduce the corresponding correlated background noise in stochastic gravitational-wave background studies. It seems natural for gravitational-wave and lightning communities to unify their efforts for mutual benefit.

\section{Acknowledgments}

The work is supported by the Ministry of Education and Science of the Russian Federation.

[1] B. P. Abbott et al. [LIGO Scientific and Virgo Collaborations], Observation of Gravitational Waves from a Binary Black Hole Merger, Phys. Rev. Lett. 116 (2016), 061102.

[2] B. P. Abbott et al. [LIGO Scientific and Virgo Collaborations], GW151226: Observation of Gravitational Waves from a 22-Solar-Mass Binary Black Hole Coalescence, Phys. Rev. Lett. 116 (2016), 241103.

[3] B. P. Abbott et al. [LIGO Scientific and VIRGO Collaborations], GW170104: Observation of a 50-Solar-Mass Binary Black Hole Coalescence at Redshift 0.2, Phys. Rev. Lett. 118 (2017), 221101. 
[4] B. P. Abbott et al. [LIGO Scientific and Virgo Collaborations], GW170814: A Three-Detector Observation of Gravitational Waves from a Binary Black Hole Coalescence, Phys. Rev. Lett. 119 (2017), 141101.

[5] B. P. Abbott et al. [LIGO Scientific and Virgo Collaborations], GW170817: Observation of Gravitational Waves from a Binary Neutron Star Inspiral, Phys. Rev. Lett. 119 (2017), 161101.

[6] B. P. Abbott et al. [LIGO Scientific and Virgo Collaborations], Characterization of transient noise in Advanced LIGO relevant to gravitational wave signal GW150914, Class. Quant. Grav. 33 (2016), 134001.

[7] B. P. Abbott et al. [LIGO Scientific and Virgo Collaborations], GW150914: First results from the search for binary black hole coalescence with Advanced LIGO, Phys. Rev. D 93 (2016) 122003.

[8] D. V. Martynov et al. [LIGO Scientific Collaboration], Sensitivity of the Advanced LIGO detectors at the beginning of gravitational wave astronomy, Phys. Rev. D 93 (2016), 112004.

[9] E. Thrane, N. Christensen, R. M. S. Schofield and A. Effler, Correlated noise in networks of gravitational-wave detectors: subtraction and mitigation, Phys. Rev. D 90 (2014), 023013.

[10] M. W. Coughlin et al., Subtraction of correlated noise in global networks of gravitational-wave interferometers, Class. Quant. Grav. 33 (2016), 224003.

[11] B. Allen and J. D. Romano, Detecting a stochastic background of gravitational radiation: Signal processing strategies and sensitivities, Phys. Rev. D 59 (1999), 102001.

[12] E. Thrane, N. Christensen and R. Schofield, Correlated magnetic noise in global networks of gravitational-wave interferometers: observations and implications, Phys. Rev. D 87 (2013), 123009.

[13] J. D. Romano and N. J. Cornish, Detection methods for stochastic gravitational-wave backgrounds: a unified treatment, Living Rev. Rel. 20 (2017), 2.

[14] I. Kowalska-Leszczynska et al., Globally coherent short duration magnetic field transients and their effect on ground based gravitational-wave detectors, Class. Quantum Grav. 34 (2017), 074002.

[15] C. Price, ELF Electromagnetic Waves from Lightning: The Schumann Resonances, Atmosphere 7 (2016), 116.

[16] A. Nickolaenko and M. Hayakawa, Schumann Resonance for Tyros: Essentials of Global Electromagnetic Resonance in the Earth's Ionosphere Cavity (Tokyo: Springer, 2014).

[17] J. Galejs, Schumann Resonances, J. Res. Nat. Bur. Stand. Section D: Radio Science 69D (1965), 1043-1055.

[18] Y. T. Tanaka et al., Detection of transient ELF emission caused by the extremely intense cosmic gamma-ray flare of 27 December 2004, Geoph. Res. Lett. 38 (2011), L08805.

[19] M. Füllekrug, Detection of thirteen resonances of radio waves from particularly intense lightning discharges, Geoph. Res. Lett. 32 (2005), L13809.

[20] B. P. Besser, Synopsis of the historical development of Schumann resonances, Radio Science 42 (2007), RS2S02.

[21] A. A. Minakov, A. P. Nikolaenko and L. M. Rabinovich, Gravitational-to-electromagnetic wave conversion in electrostatic field of earth-ionosphere resonator, Radiophys. Quant. Electron. 35 (1997), 318-323.

[22] R. I. Albrecht et al., Where are the lightning hotspots on earth? Bull. Am. Meteorol. Soc. 1 (2016), $2051-2068$.

[23] R. W. Sinnott, Virtues of the Haversine, Sky and Telescope 68N2 (1984), 159-170.

[24] B. S. Shylaja, From navigation to star hopping: forgotten formulae, Resonance 20N4 (2015), 352-359.

[25] T. Ogawa and M. Komatsu, Analysis of Q burst waveforms, Radio Science 42 (2007), RS2S18.

[26] T. Ogawa and M. Komatsu, Propagation velocity of VLF EM waves from lightning discharges producing Q-bursts observed in the range 10-15 Mm, Atmospheric Research 95 (2010), 101-107.

[27] I. Silber, C. Price, E. Galanti and A.Shuval Anomalously strong vertical magnetic fields from distant ELF/VLF sources, J. Geoph. Res. Space Phys. 120 (2015), 6036-6044.

[28] E. Williams et al., The Relationship Between the Background and Transient Signals in Schumann Resonances, NASA/CP1999-209261 (Proceedings of the 11th International Conference on Atmospheric Electricity), pp. 758-761.

[29] A. Guha et al., Aliasing of Schumann resonance background signal by sprite-associated Q-bursts, Journal of Atmospheric and Solar-Terrestrial Physics 165-166 (2017), 25-37. 